\title{
Collagen-chitosan scaffold modified with Au and Ag nanoparticles: Synthesis and structure
}

\author{
M.S. Rubina ${ }^{\text {a }}$, E.E. Kamitov ${ }^{\text {a }}$, Ya. V. Zubavichus ${ }^{\text {b }}$, G.S. Peters ${ }^{b}$, A.V. Naumkin ${ }^{a}$, S. Suzer ${ }^{c}$, \\ A.Yu. Vasil'kov ${ }^{\mathrm{a}, *}$ \\ a A.N. Nesmeyanov Institute of Organoelement Compounds, Russian Academy of Sciences, Moscow, 119991 Russian Federation \\ b National Research center «Kurchatov Institute», Moscow, 123182 Russian Federation \\ c Department of Chemistry, Bilkent University, Ankara, 06800 Turkey
}

\section{A R T I C L E I N F O}

\section{Article history:}

Received 7 May 2015

Received in revised form

28 December 2015

Accepted 13 January 2016

Available online 14 January 2016

\section{Keywords:}

Collagen-chitosan scaffolds

Metal-vapor synthesis

Nanoparticles

XPS

XRD

SAXS

XANES/EXAFS

\begin{abstract}
A B S T R A C T
Nowadays, the dermal biomimetic scaffolds are widely used in regenerative medicine. Collagen-chitosan scaffold one of these materials possesses antibacterial activity, good compatibility with living tissues and has been already used as a wound-healing material. In this article, collagen-chitosan scaffolds modified with $\mathrm{Ag}$ and Au nanoparticles have been synthesized using novel method - the metal-vapor synthesis. The nanocomposite materials are characterized by XPS, TEM, SEM and synchrotron radiation-based X-ray techniques. According to XRD data, the mean size of the nanoparticles (NPs) is $10.5 \mathrm{~nm}$ and $20.2 \mathrm{~nm}$ in Au-Collagen-Chitosan (Au-CollCh) and Ag-Collagen-Chitosan (Ag-CollCh) scaffolds, respectively in fair agreement with the TEM data. SAXS analysis of the composites reveals an asymmetric size distribution peaked at $10 \mathrm{~nm}$ for Au-CollCh and $25 \mathrm{~nm}$ for Ag-CollCh indicative of particle's aggregation. According to SEM data, the metal-carrying scaffolds have layered structure and the nanoparticles are rather uniformly distributed on the surface material. XPS data indicate that the metallic nanoparticles are in their unoxidized/neutral states and dominantly stabilized within the chitosan-rich domains.
\end{abstract}

(C) 2016 Elsevier B.V. All rights reserved.

\section{Introduction}

Naturally occurring polymers are widely applied in medicine due to their pronounced biocompatibility, availability of renewable sources, easiness of chemical modification, etc. [1]. Among them, chitosan is of special importance, which is essentially an $\mathrm{N}$-deacetylated chitin derivative, a linear polymer composed of Dglucosamine and $\mathrm{N}$-acetylglucosamine residues. Chitosan is known to promote skin regeneration, wounds and burns healing. Furthermore, it manifests hemostatic and immunomodulatory properties [2-4], as well as antibacterial and antifungal activity [5,6]. Chitosan is a biocompatible polymer and possesses a high sorption capacity. Apart from pristine chitosan, chitosan-based hybrid materials, especially ones with collagen [7], cellulose [8], polyethylene glycol [9], polyvinylpyrrolidone [10], gelatin [11], polyvinyl alcohol [12], etc., are promising materials for biomedical applications [13].

Collagen is a structural protein actively used in medicine [14]. Availability of hydroxylic groups and amino acid residues on its surface makes it possible to adjust its surface charge to either negative

\footnotetext{
* Corresponding author. Tel.: +7 4991359380.

E-mail address: alexandervasilkov@yandex.ru (A.Yu. Vasil'kov).
}

or positive values by changing $\mathrm{pH}$ [15]. Collagen fibrils (crosslinked or not, native or denaturated) strongly affect morphology and physiology of cells [16]. Collagen is nearly as biodegradable and biocompatible as chitosan, that is why it is widely used in tissue engineering as wound and burn dressing and sponges. Collagen and chitosan do not occur in nature together, but specific properties of both polymers can be utilized to design a hybrid material with unique structural and mechanical properties [17].

Numerous examples of similar materials used for the tissue engineering, drug delivery matrices, bandage dressing, etc., are available in literature $[18,19]$. Incorporation of noble metal NPs with antibacterial activity further extends approved fields of applications of collagen- chitosan scaffolds. The majority of methods for the incorporation of metal NPs in polymer matrices requires chemical reduction of metal salts impregnated into the polymer matrix (e.g., borohydride or citrate methods) [20,21]. These techniques are typically multi-step and use potentially biologically hazardous or environmentally unfriendly reactants, stabilizers, or reducing agents [22], which prevent or strongly limit the use of the resultant composites in medicine [23].

Metal-vapor synthesis (MVS) is an efficient route to produce biologically active metal nanoparticles and the composites derived from them with biocompatible materials for biomedical 
applications $[24,25]$. The technique has been successfully applied to modify common medical materials, including surgical suture and dressing materials or implants, with metal nanoparticles possessing antibacterial and antifungal property [26,27]. In contrast to the majority of methods for preparation of nanoparticles, the MVS method is fully environmentally safe and can easily be integrated into diverse technological cycles. The MVS method affords colloidal suspensions of NPs in common medical solvents, which makes any further purification unnecessary [28]. The target composites are prepared via the modification of a biopolymer with organosols containing metal nanoparticles followed by solvent removal.

Here, we report for the first time on the synthesis of metal-bearing hybrid systems based on the intrinsically porous collagen-chitosan scaffolds. The structure and chemical states of metals in the composite nanomaterials are addressed by modern physicochemical techniques.

\section{Experimental}

\subsection{Materials}

Collagen-chitosan scaffold, denoted as CollCh, was prepared according to the method described elsewhere [29]. $\mathrm{Et}_{3} \mathrm{~N}$ (Sigma Aldrich, purity $\geq 99.5 \%$ ) and i-PrOH (Fluka, purity 99.8\%) were used as organic solvents. All other reactants used for the experiments were of analytical grade. Prior to use in the synthesis, all solvents were dried, distilled in an atmosphere of purified $\mathrm{Ar}$ and degassed by several consecutive pump-freeze-thaw cycles at $10^{-1} \mathrm{~Pa}$ and RT for $1 \mathrm{~h}$. The resultant collagen-chitosan scaffold was degassed in vacuo.

\subsection{Metal-vapor synthesis of hybrid materials}

The original metal-vapor synthesis (MVS) method was used in this work to produce metal-modified composites based on the collagen-chitosan scaffold. The (MVS) method is efficient in preparation of highly reactive metal nanoparticles and their incorporation into various matrices to induce practically important magnetic [30], antibacterial [26], catalytic [31] and tribological [32] properties.

Nanocomposites filled with gold and silver NPs were prepared by impregnation of the metal-containing organosols from MVS into the collagen-chitosan scaffold as described elsewhere [26]. Metals were evaporated at a base pressure of $10^{-2} \mathrm{~Pa}$ by a resistively heated evaporator in the form of either tungsten rod or small tantalum vessel for $\mathrm{Au}$ (99.99\%) or $\mathrm{Ag}$ (99.99\%), respectively. Specially preconditioned organic solvents $\mathrm{Et}_{3} \mathrm{~N}$ and $\mathrm{i}-\mathrm{PrOH}$ were used to stabilize, respectively, Au and Ag nanoparticles as sols. Metal vapor was condensed simultaneously with the solvent vapor onto liquid nitrogen-cooled walls of a glass reactor with a volume of $5 \mathrm{~L}$. A typical solvent-to-metal molar ratio in the synthesis was 300:1. After the synthesis, the co-condensate was heated to the melting point and the resultant organosol was impregnated into the collagen-chitosan scaffold placed in a Schlenk flask under vacuum. The excessive amount of the organosol was removed and the target product was dried in vacuum at $60^{\circ} \mathrm{C}$.

\subsection{Characterization methods}

\subsubsection{Synchrotron radiation-based techniques}

X-ray structural studies of the composites based on collagenchitosan scaffolds modified with Au and Ag nanoparticles, including such techniques as powder X-ray diffraction, small- angle X-ray scattering, and X-ray absorption spectroscopy XANES/EXAFS were performed at the Kurchatov synchrotron radiation source (NRC "Kurchatov Institute", Moscow).
X-ray absorption spectra and powder diffraction patterns were measured at the Structural Materials Science beamline [33]. Au $\mathrm{L}_{3}$-edge XANES/EXAFS for the gold-containing sample Au-CollCh as well as for the Au foil reference were measured in the transmission mode using ion chambers filled with appropriate $\mathrm{N}_{2}-\mathrm{Ar}$ mixtures. For similar Ag-containing composites, the silver concentration appeared insufficient for transmission measurements and thus the spectra were measured in the X-ray fluorescence yield mode using a Si avalanche photodiode. Spectra for the Ag foil reference were measured in the transmission mode. Experimental data processing and analysis were performed using the IFEFFIT software package [34].

Powder X-ray diffraction patterns for the same samples were measured in the transmission (Debye-Sherrer) mode using Fuji Film Imaging plates as a 2D detector. Diffraction measurements were performed at an X-ray wavelength $\lambda=0.06889 \mathrm{~nm}$. The mean nanoparticle size was estimated by profile analysis assuming the Pseudo-Voight line shape of the diffraction peaks with instrumental function responsible for the Gaussian part of broadening and Lorentzian-type sample-driven physical broadening.

Additionally, small-angle X-ray scattering curves were measured for the pristine and Ag-, Au-filled collagen-chitosan scaffold to refine the diffraction data on NP sizing. The measurements were performed at the DICSI beamline of the same synchrotron source and a 2D MAR165 CCD detector was used. The sample-todetector distance was $2400 \mathrm{~mm}$ and the X-ray wavelength was set to $\lambda=0.162 \mathrm{~nm}$. To improve sampling and signal-to-noise statistics, several data sets at different spots on a sample were measured and averaged. The size distribution of $\mathrm{Ag}$ and $\mathrm{Au}$ nanoparticles was retrieved from the corresponding SAXS "Ag-CollCh - CollCh" and "Au-CollCh-CollCh" difference curves, respectively. The indirect Fourier transformation approach as implemented in the GNOM code under assumption of a polydisperse distribution of noninteracting hard spheres [35] has been used.

\subsubsection{X-ray photoelectron spectroscopy (XPS)}

X-ray photoelectron spectra were acquired with an Axis Ultra DLD (Kratos, UK) spectrometer using $\mathrm{Al} \mathrm{K}_{\alpha}$ radiation at an operating power of $150 \mathrm{~W}$ of the X-ray tube. Survey and high- resolution spectra of appropriate core levels were recorded at pass energies of $160 \mathrm{eV}$ and $40 \mathrm{eV}$ and with scanning steps of $1 \mathrm{eV}$ and $0.1 \mathrm{eV}$, respectively. Sample area of $300 \mu \mathrm{m} \times 700 \mu \mathrm{m}$ contributed to the spectra. The samples were mounted on a sample holder with a two-sided adhesive tape, and the spectra were collected at room temperature. The base pressure in the analytical UHV chamber of the spectrometer during measurements did not exceed $10^{-8}$ Torr. The binding energy scale of the spectrometer was calibrated to provide the following values for reference samples (i.e., metal surfaces freshly cleaned by ion sputtered): $\mathrm{Au} 4 \mathrm{f}_{7 / 2}-83.96 \mathrm{eV}, \mathrm{Cu} 2 \mathrm{p}_{3 / 2}-932.62 \mathrm{eV}$, $\mathrm{Ag} 3 \mathrm{~d}_{5 / 2}-368.21 \mathrm{eV}$. The electrostatic charging effects were compensated by using an electron neutralizer. For each sample, the spectra were recalibrated against the adventitious carbon signal at $285.0 \mathrm{eV}$. Background with inelastic losses was subtracted from the high-resolution spectra according to the Shirley prescription. The Ag MNN Auger spectra were corrected using a linear background. The surface chemical composition was calculated using standard element sensitivity factors coded in the spectrometer control software corrected for the transfer function of the instrument.

\subsubsection{TEM}

Micrographs of the samples were made using a transmission electron microscope LEO 912AB OMEGA, Zeiss (Germany).

\subsubsection{SEM}

SEM analysis was performed with a scanning electron microscope Tescan Mira LMU (Czech Republic). The samples were fixed 


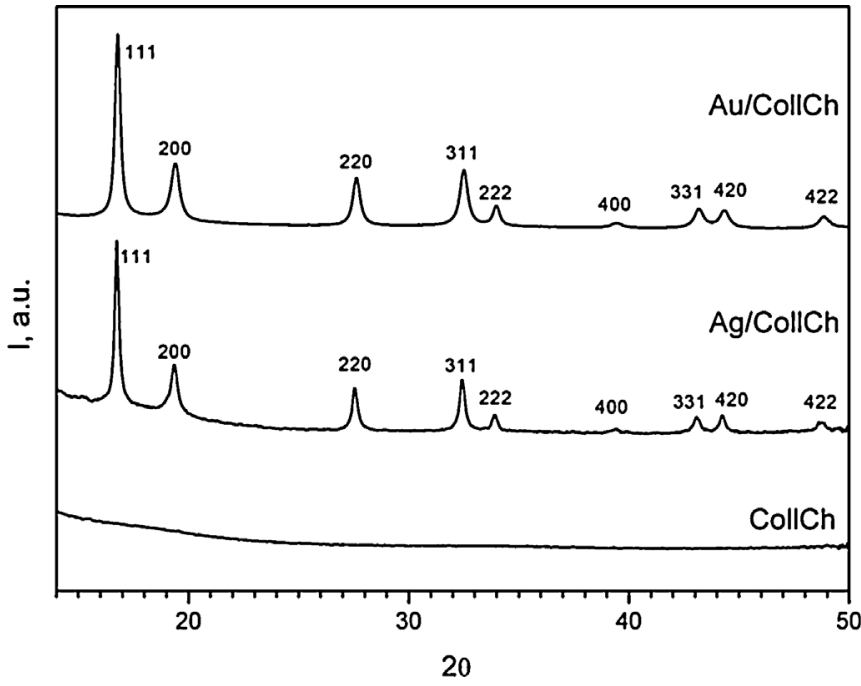

Fig. 1. XRD patterns for the nanocomposites studied $\mathrm{Au}-\mathrm{CollCh}, \mathrm{Ag}-\mathrm{CollCh}$, and pristine CollCh $(\lambda=0.06889 \mathrm{~nm})$.

on a conductive tape and examined under high vacuum with the Everhart-Thornley standard secondary electron detector.

\section{Results and discussion}

The experimental X-ray diffraction patterns of the collagenchitosan scaffolds modified by gold and silver nanoparticles are shown in Fig. 1. The collagen-chitosan scaffold appears to be amorphous so that all diffraction peaks present in the respective patterns can be attributed to fcc atomic packing of the Ag and Au nanoparticles [PDF \#040783 and \#040784, respectively]. The crystallite sizes estimated from the line broadening analysis assuming that microstrains make negligible contribution yield $10.5 \mathrm{~nm}$ and $20.2 \mathrm{~nm}$ for $\mathrm{Au}-\mathrm{CollCh}$ and $\mathrm{Ag}$-CollCh, respectively, the nominal accuracy of the estimate is $10-15 \%$.

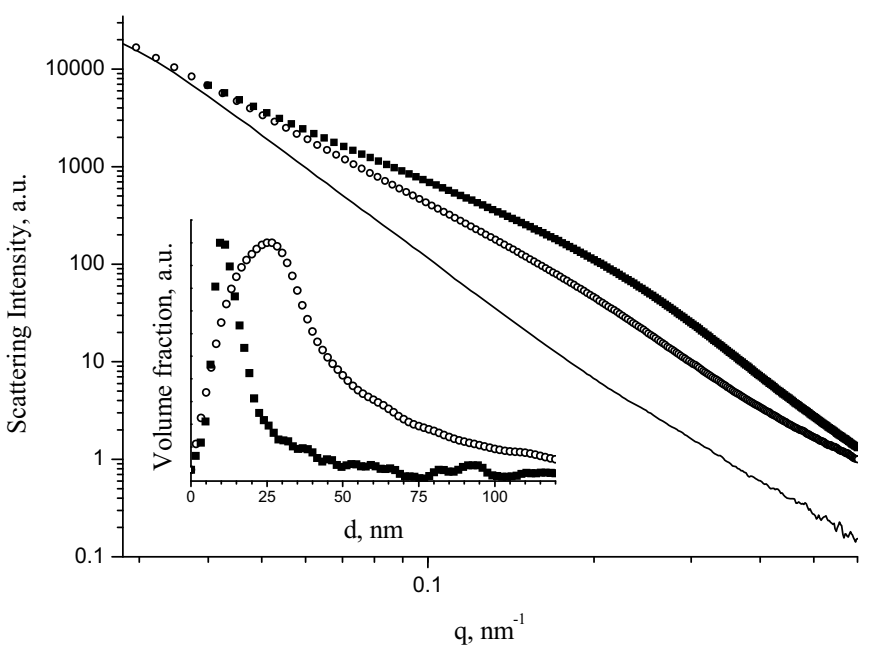

Fig. 2. Experimental SAXS curves for Au-CollCh (filled squares), Ag-CollCh (open circles), and pristine CollCh (line). The inset shows volume distribution of metal nanoparticle diameters reconstructed from the difference curves.

For the composites, an independent estimate for the nanoparticles size was obtained from the SAXS data. The corresponding experimental data are shown in Fig. 2.

The experimental curve for the metal-containing composite (corrected for the incident intensity and the sample X-ray absorption) is characterized by a higher scattering intensity than the pristine CollCh scaffold over the entire range of scattering wave vectors measured, which makes it possible to reliably separate the contribution of the scattering by the nanoparticles via simple numerical subtraction procedure. An analysis of the difference curve using the indirect Fourier transformation with the Tikhonov's regularization under assumption of none-interacting hard spheres yields a rather narrow and slightly asymmetric size distribution peaked at $10 \mathrm{~nm}$ for Au-CollCh and much broader asymmetric distribution curve with a maximum at $25 \mathrm{~nm}$ extending up to sizes
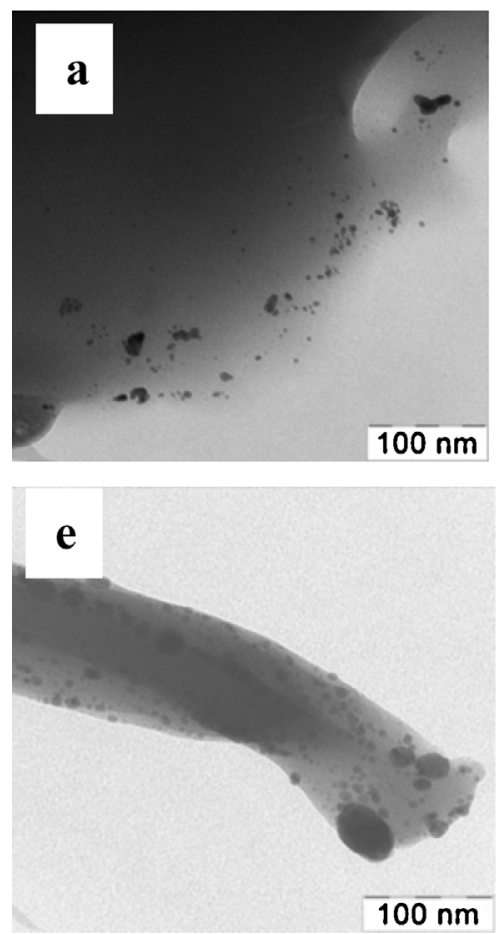
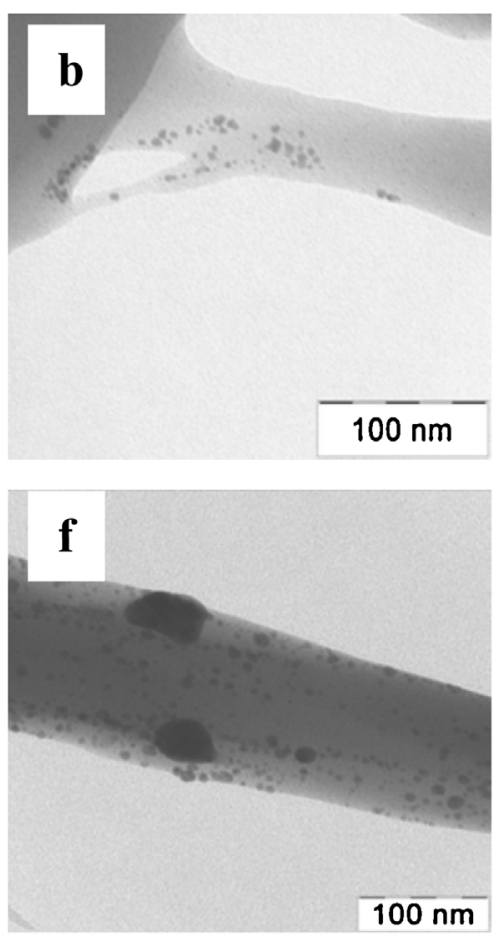
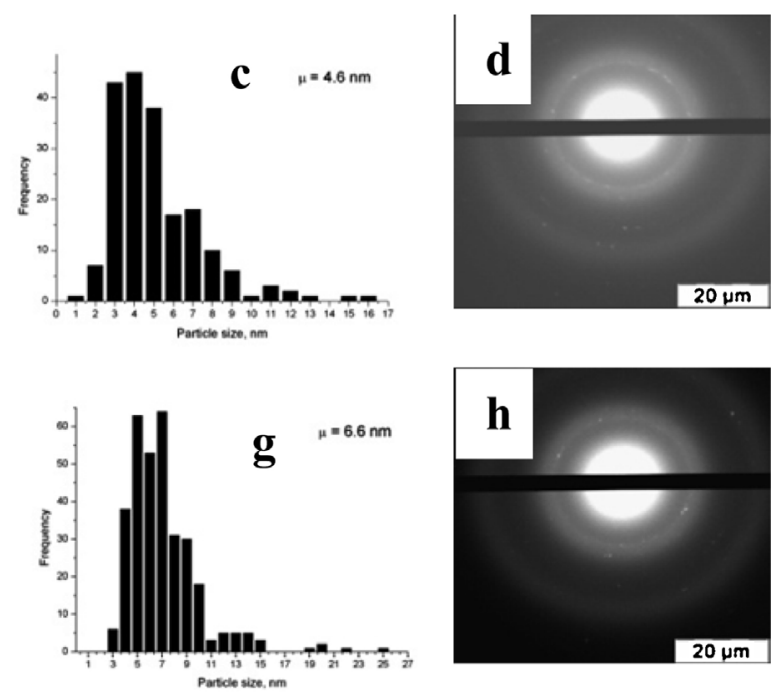

Fig. 3. TEM micrographs (scale bar is $100 \mathrm{~nm}$ ), corresponding SAED patterns and particle size distribution for the Au-CollCh (a-d) and Ag-CollCh (e-h) composites. 

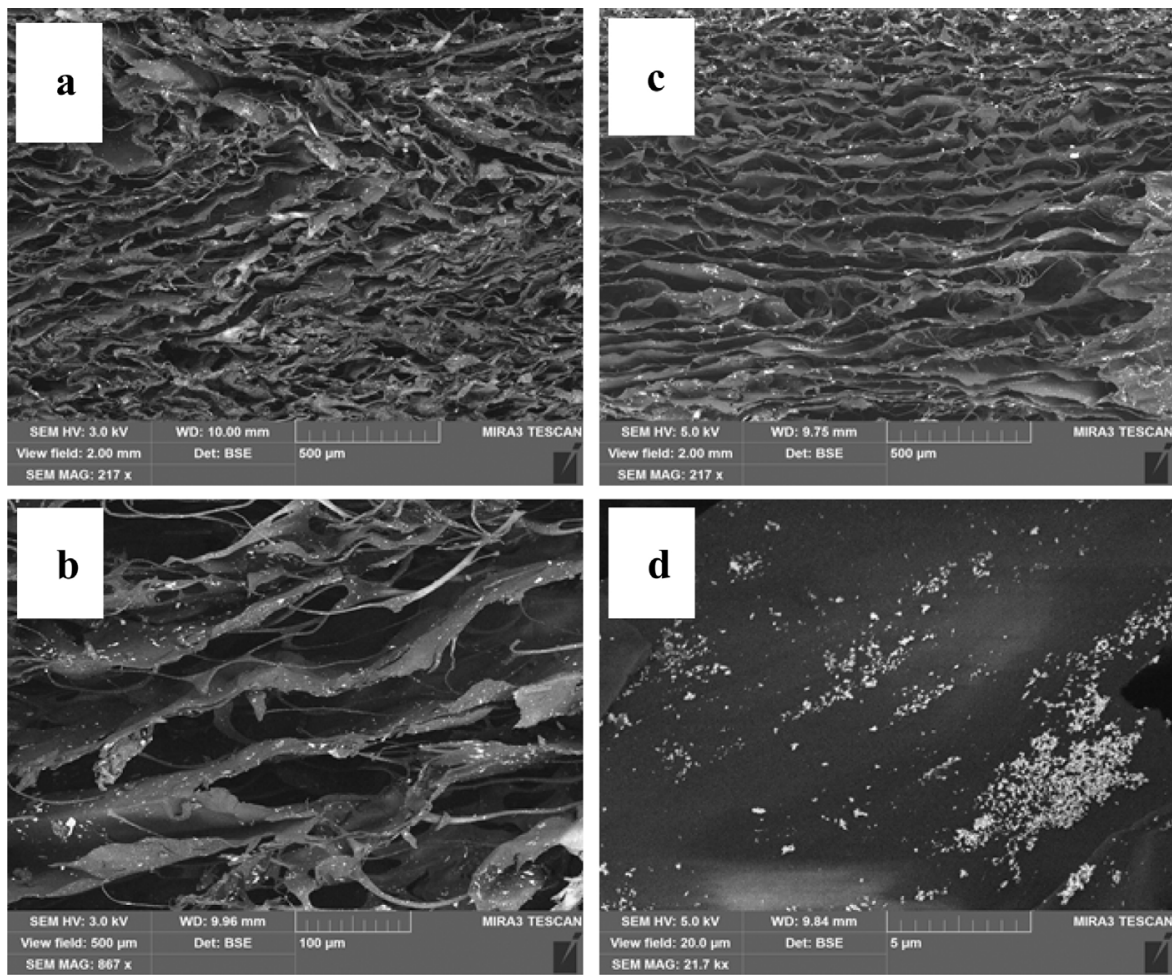

Fig. 4. SEM micrographs of the cross-section of the Au-CollCh (a, b) and Ag-CollCh (c, d) composites.

of $120-150 \mathrm{~nm}$ for Ag-CollCh. Apparently, this means that the nanoparticles are agglomerated into aggregates from few to several thousand individual nanoparticles within the composite.

Fig. 3 shows micrographs, SAED patterns of metal nanoparticles and size distribution histogram for the Au-CollCh and Ag-CollCh. According to TEM, the particles are spherical, polydisperse and have fairly uniform distribution in the scaffold. The average size of the Au and Ag particles is $4.6 \mathrm{~nm}$ and $6.6 \mathrm{~nm}$, respectively. It is demonstrated in Fig. 3(c and g) that Ag-CollCh composite have the broader particle size distribution than Au-CollCh one.

The presence of larger $\mathrm{Ag}$ nanoparticles with size of about $60-70 \mathrm{~nm}$ in the material shown in Fig. 3(e and f) can be explained by aggregation of smaller nanoparticles during the modification of polymer matrix with organosol, as well as surface diversity of the pristine scaffold.

Fig. 4 shows the internal cross-sectional microstructure of the $\mathrm{Ag}$-CollCh and Au-CollCh composites. Collagen-chitosan scaffold has a layered structure, consisting of lamellas and microcavaties formed by polymer network of fibrils (Fig. $4 a-c$ ).
The average diameter of microcavities is about $40-80$ microns and the thickness of the fibril scaffold is $2-5$ microns. It is exhibited that the aggregates, with sizes from a few tens of nanometers to several microns, have slightly uniform distributions on the surfaces of the fibrils and lamellas (Fig. 4b and d).

Supplementary information on the electronic state and local environment of $\mathrm{Au}$ and $\mathrm{Ag}$ atoms in the composites was obtained by X-ray absorption spectroscopy XANES/EXAFS. Au L $\mathrm{L}_{3}$ - and Ag K-edge XANES spectra for the metal-modified CollCh-based composites are compared with reference spectra of metal foils in Fig. 5.

The similar shape and energy position of EXAFS spectral features indicate that the chemical state of the metal atoms in the composites is similar to that in their bulk metals, which means that the fraction of surface atoms strongly interacting with the matrix is rather low.

For the XANES spectrum of the Ag-CollCh composite, an increase in the period of oscillations is apparent, which corresponds to an $\mathrm{Ag}-\mathrm{Ag}$ bond length contraction with respect to the bulk metal. This finding is further supported by a quantitative analysis of the EXAFS
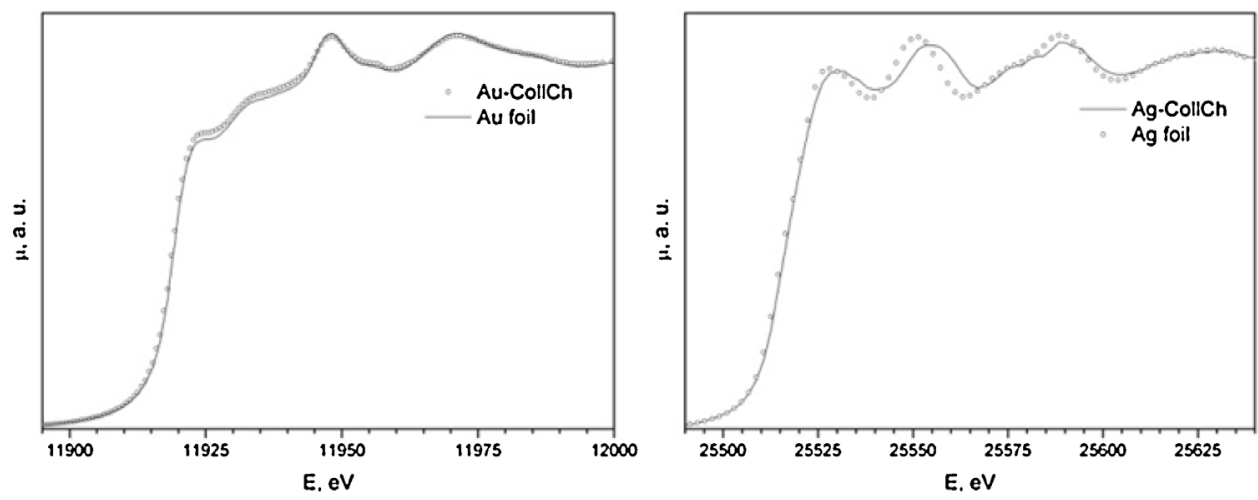

Fig. 5. XANES spectra of collagen-chitosan scaffold modified with Au (left) and Ag (right). 

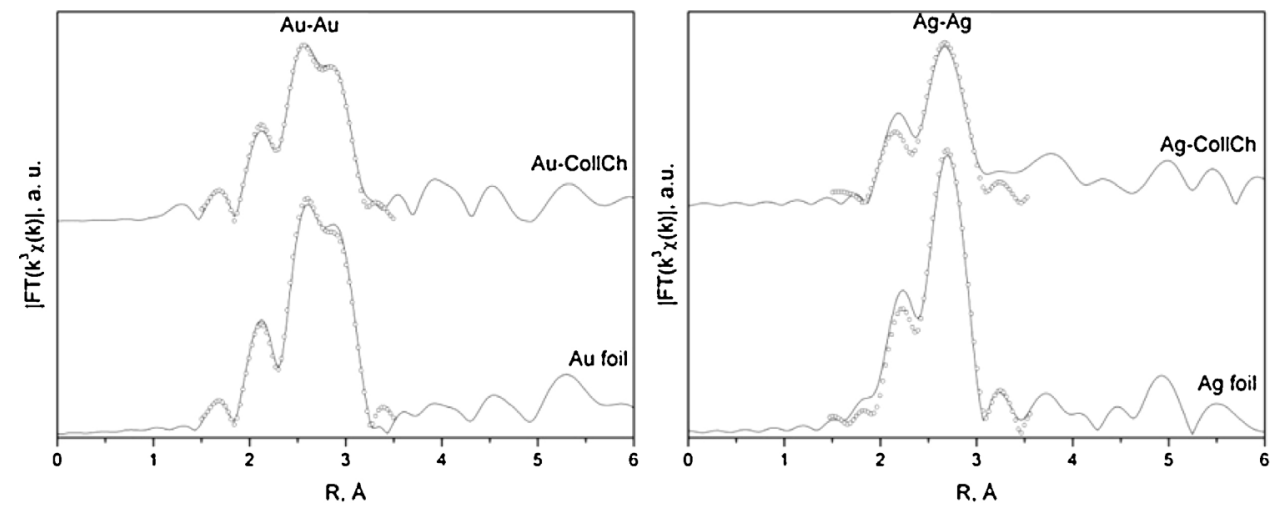

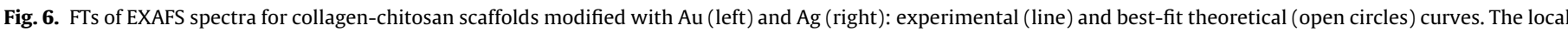
environment parameters corresponding to the fits are summarized in Table 1.

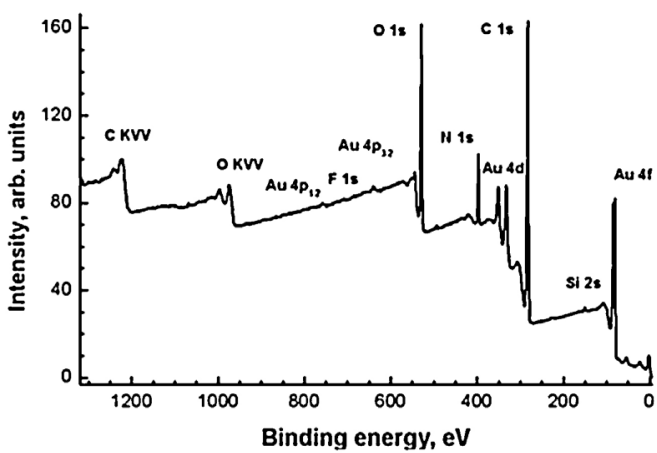

a

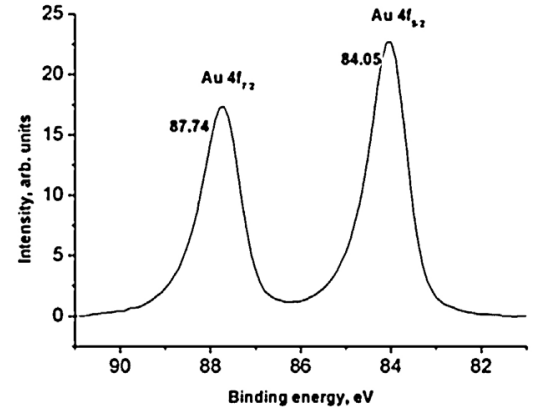

c

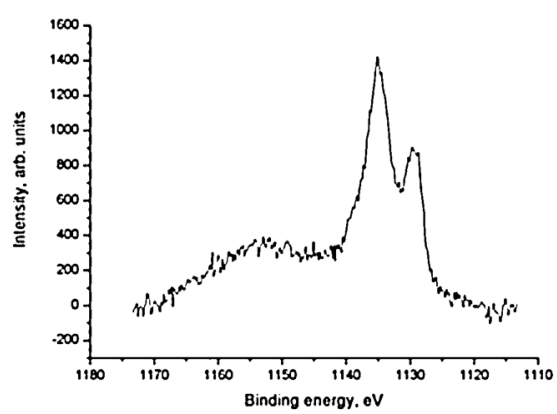

e

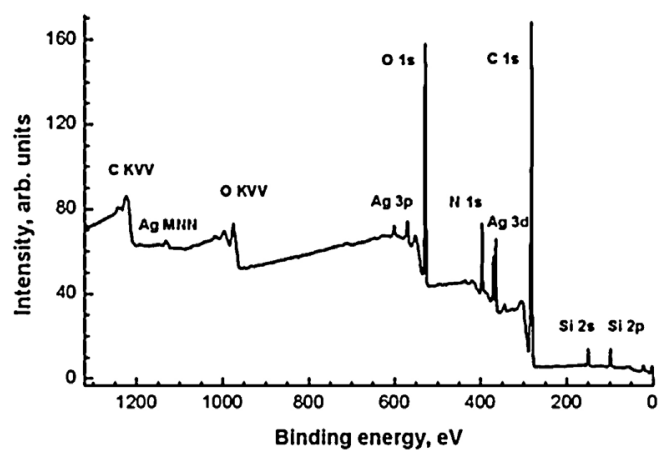

b

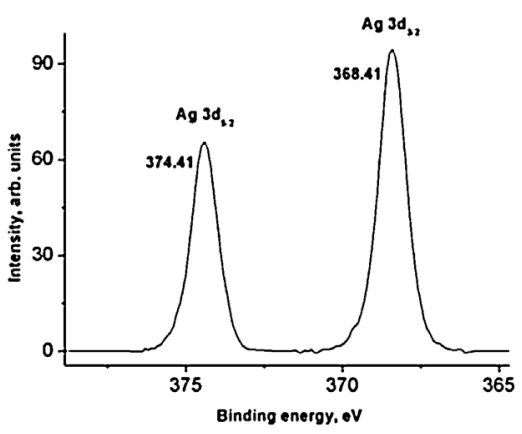

d

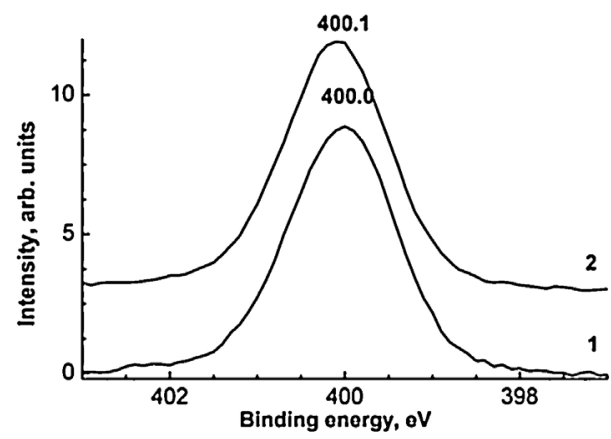

f

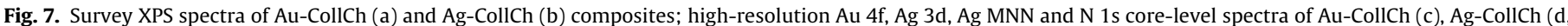
e) and (f1, f2), respectively. 
Table 1

Parameters of the local environment of the metal atoms in the chitosan-based composites according to EXAFS: interatomic distances $(R)$, coordination numbers $(n)$, and Debye-Waller factors $\left(\sigma^{2}\right)$

\begin{tabular}{lllll}
\hline Sample & Path & $n$ & $R[\AA]$ & $\sigma^{2}\left[\AA^{2}\right]$ \\
\hline Au (foil) & Au-Au & 12.0 & 2.85 & 0.0079 \\
Au-CollCh & Au-Au & 11.9 & 2.84 & 0.0095 \\
Ag (foil) & Ag-Ag & 12.0 & 2.88 & 0.0095 \\
Ag-CollCh & Ag-Ag & 9.7 & 2.85 & 0.0119 \\
\hline
\end{tabular}

data. Fourier Transforms (FTs) of EXAFS spectra for the composites under study are shown in Fig. 6. They are essentially similar to those of the reference metal foils. Best-fit coordination numbers and interatomic distances for the first metal-metal coordination spheres are listed in Table 1.

The composites are characterized by somewhat decreased coordination numbers when compared with the data the for bulk metals, which is very common for nanometer-sized metal particles. The minimum metal-metal coordination number is observed for the Ag-CollCh composite despite its rather larger crystallite size as estimated from diffraction data. Furthermore, the $\mathrm{Ag}-\mathrm{Ag}$ interatomic distance derived from the EXAFS for that composite is by $0.03 \AA$ shorter than that for $\mathrm{Ag}$ foil. The bondlength contraction is quite typical of small metal nanoparticles. But in the case of silver, bondlength elongation is sometimes observed due to partial oxidation and suboxide formation [36]. Nevertheless, a prominent change in the coordination number and bondlength for the AgCollCh despite nominally large size from diffraction may indicate a distorted local structure of silver nanoparticles with frequent point defects.

In order to evaluate the surface concentrations and chemical states of metal atoms in the composites (within a layer of 5-8 nm), $\mathrm{X}$-ray photoelectron spectroscopy was applied (Fig. 7). Survey spectra of Au-CollCh (Fig. 7a) and Ag-CollCh (Fig. 7b) reveal peaks attributable to elements of the nominal chemical composition, i.e., C, O, N, Au/Ag, as well as admixture elements Si and F. Quantification data based on atomic sensitivity factors are presented in Table 2. As it can be judged from $\mathrm{C} / \mathrm{N}$ and $\mathrm{C} / \mathrm{O}$ atomic ratios, the incorporation of metals into the pristine collagen-chitosan scaffolds gives rise to some enrichment of the composite surfaces with carbon species (see Table 2). Partly, this can be due to co-sorption of the organic solvent used in the synthesis. The absence of dramatic changes in element concentrations rather confirms that the CollCh scaffolds do not degrade upon modification with noble metal nanoparticles.

The $\mathrm{Au} 4 \mathrm{f}$ binding energy observed for the composite (Fig. 7c) is characteristic of the $\mathrm{Au}^{0}$ state. A minute shift by $0.05 \mathrm{eV}$ to a higher energy with respect to foil and pronounced asymmetry of the peak shape are due to the size effects $[37,38]$ and thus they also confirm the presence of nm-sized gold nanoparticles therein.

High-resolution Ag 3d core-levels and MNN Auger lines for the Ag-containing composite are shown in Fig. $7 d$ and e. The experimental values for the Ag 3d binding energy, Ag MNN Auger kinetic energy, and the corresponding Auger parameter all confirm [39,40] the $\mathrm{Ag}^{0}$ state of silver atoms in the composite. The $\mathrm{N} 1 \mathrm{~s}$ spectra in metal-containing composites and pristine scaffolds are characterized by different binding energies and full-widths at half-maxima

Table 2

Surface chemical compositions of the composites from XPS data.

\begin{tabular}{lllllllll}
\hline Sample & \multicolumn{7}{l}{ Relative concentration, at. \% } \\
\cline { 2 - 9 } & $\mathrm{C}$ & $\mathrm{N}$ & $\mathrm{O}$ & $\mathrm{Au}$ & $\mathrm{Ag}$ & $\mathrm{C} / \mathrm{N}$ & $\mathrm{C} / \mathrm{O}$ & $\mathrm{O} / \mathrm{N}$ \\
\hline CollCh & 74.2 & 8.5 & 17.3 & - & - & 8.7 & 4.3 & 2.0 \\
Au-CollCh & 74.0 & 7.8 & 15.8 & 2.4 & - & 9.5 & 4.7 & 2.0 \\
Ag-CollCh & 75.2 & 8.1 & 15.8 & - & 1.0 & 9.3 & 4.8 & 2.0 \\
\hline
\end{tabular}

(Fig. 7f), which can be explained by an alteration of balance between the collagen and chitosan $\mathrm{N}$-functional groups upon the modification. Typical $\mathrm{N} 1 \mathrm{~s}$ core-level binding energies in chitosan $\left(\mathrm{C}-\mathrm{NH}_{2}\right)$ and collagen $(\mathrm{C}(\mathrm{O}) \mathrm{N})$ are $399.67 \mathrm{eV}$ [41] and 399.77-399.96 [42], respectively.

\section{Conclusions}

The novel method for the synthesis of hybrid materials based on the collagen-chitosan scaffold modified with $\mathrm{Ag}$ and $\mathrm{Au}$ nanoparticles is reported. XRD and SAXS data show that the Au- and Ag-bearing composites are characterized by mean particle size of $10 \mathrm{~nm}$ and $25 \mathrm{~nm}$, respectively. It is revealed that nanoparticles in the bulk of materials have a slightly uniform distribution with the mean size is $4.6 \mathrm{~nm}$ and $6.6 \mathrm{~nm}$ for $\mathrm{Au}$ and $\mathrm{Ag}$, respectively. On the surface it was observed the larger aggregates, with sizes from a few tens of nanometers to several microns, consisting of smaller particles. The whole structure of the obtained nanocomposites is similar. However, the Ag-CollCh composite has much broader asymmetric size distribution than Au-CollCh.

According to XPS, metal atoms are in the unoxidized/neutral states and preferably stabilized in the chitosan matrix. Similarly, XANES/EXAFS data indicate the chemical state and local structure of metal atoms in the composites is similar to that of bulk metals apart from a small decrease in metal-metal coordination number in both composites, and metal-metal bond-length contraction in Ag-CollCh.

We suggest that collagen-chitosan scaffold, containing Au and Ag nanoparticles, can be promising antibacterial wound-healing material for medicine.

\section{Acknowledgements}

This work was partially supported by the Russian Foundation for Basic Research (grants nos. 14-03-01074 and 15-53-61030).

\section{References}

[1] A. Aravamudhan, D.M. Ramos, A.A. Nada, S.G. Kumbar, Natural polymers: polysaccharides and their derivatives for biomedical applications, in: S. Kumbar, C. Laurencin, M. Deng (Eds.), Natural and Synthetic Biomedical Polymers, first ed., Elsevier, USA, 2014, pp. 67-89.

[2] R. Jayakumar, M. Prabahran, P.T. Sudheesh Kumar, S.V. Nair, H. Tamura, Biomaterials based on chitin and chitosan in wound dressing applications, Biotechnol. Adv. 29 (2011) 322-337.

[3] S.-Y. Ong, J. Wu, S.M. Moochhala, M.-H. Tan, J. Lu, Development of a chitosan-based wound dressing with improved hemostatic and antimicrobial properties, Biomaterials 29 (2008) 4323-4332.

[4] A. Pattani, V.B. Patravale, L. Panicker, P.D. Potdar, Immunological effects and membrane interactions of chitosan nanoparticles, Mol. Pharm. 6 (2009) 345-352.

[5] I. Younes, S. Sellimi, M. Rinaudo, K. Jellouli, M. Nasri, Influence of acetylation degree and molecular weight of homogeneous chitosans on antibacterial and antifungal activities, Int. J. Food Microbiol. 185 (2014) 57-63.

[6] J. Xu, X. Zhao, X. Han, Y. Du, Antifungal acivity og oligochitosan against Phytophtora capsici and other plant pathogenic fungi in vitro, Pest. Biochem. Physiol. 87 (2007) 220-228.

[7] J.-P. Chen, G.-Y. Chang, J.-K. Chen, Electrospun collagen/chitosan nanofibrous membrane as wound dressing, Coll. Surf. A: Physicochem. Eng. Asp. 313-314 (2008) 183-188.

[8] M.I. Niyas Ahamed, S. Sankar, P. Mohammed Kashif, S.K. Hayath Basha, T.P. Sastry, Evaluation of biomaterial containing regenerated cellulose and chitosan incorporated with silver nanoparticles, Int. J. Biol. Macromol. 72 (2015) 680-686.

[9] C. Prego, D. Torres, E. Fernandez-Megia, R. Novoa-Carballal, E. Quiñoá, M.J. Alonso, Chitosan-PEG nanocapsules as new carriers for oral peptide delivery: effect of chitosan pegylation degree, J. Controlled Release 111 (2006) 299-308.

[10] T. Çaykara, A. Alaslan, M.S. Eroğlu, O. Güven, Surface energetics of poly(N-vinyl-2- pyrrolidone)/chitosan blend films, Appl. Surf. Sci. 252 (2006) $7430-7435$.

[11] C.-M. Deng, L.-Z. He, M. Zhao, D. Yang, Y. Liu, Biological properties of the chitosan- gelatin sponge wound dressing, Carbohydr. Polym. 69 (2007) $583-589$. 
[12] E. Salehi, S.S. Madaeni, Influence of poly(ethylene glycol) as pore-generator on morphology and performance of chitosan/poly(vinyl alcohol) membrane adsorbents, Appl. Surf. Sci. 288 (2014) 537-541.

[13] M. Rinaudo, Chitin and Chitosan: properties and applications, Prog. Polym. Sci. 31 (2006) 603-632

[14] M. Chvapil, R.L. Kronenthal, W.V. Winkle Jr., Medical and surgical application of collagen, Int. Rev. Connective Tissue Res. 6 (1973) 1-61.

[15] T. Nezu, F.M. Winnik, Interaction of water-soluble collagen with poly(acrylic acid), Biomaterials 21 (2000) 415-419.

[16] K. Yoshizato, A. Makino, K. Nagayoshi, Regulation of morphology and physiology of epithelial cells by collagen fibrils, Biomed. Res. 9 (1988) 33-45.

[17] T. Garg, O. Singh, S. Arora, R.S.R. Murthy, Scaffold. A novel carrier for cell and drug delivery, Crit. Rev. Therap. Drug Carrier Syst. 29 (2012) 1-63.

[18] X.H. Wang, D.P. Li, W.J. Wang, Q.L. Feng, F.Z. Cui, Y.X. Xu, X.H. Song, M. van de Werf, Crosslinked collagen/chitosan matrix for artificial livers, Biomaterials 24 (2003) 3213-3220.

[19] L. Ma, C. Gao, Z. Mao, J. Zhou, J. Shen, X. Hu, C. Han, Collagen/chitosan porous scaffolds with improved biostability for skin tissue engineering, Biomaterials 24 (2003) 4833-4841.

[20] L. -Ping Ding, Y. Fang, An investigation of the surface-enhanced Raman scattering (SERS) effect from laser irradiation of Ag nanoparticles prepared by trisodium citrate reduction method, Appl. Surf. Sci. 253 (2007) 4450-4455.

[21] W. Zhanga, X. Qiao, Q. Chen, Y. Cai, H. Chen, The influence of synthesis condition and aging process of silver nanocrystals on the formation of silver nanorods, Appl. Surf. Sci. 258 (2012) 5909-5913.

[22] D. Wei, W. Sun, W. Qian, Y. Ye, X. Mac, The synthesis of chitosan-based silver nanoparticles and their antibacterial activity, Carbohydr. Res. 344 (2009) 2375-2382.

[23] Y.-K. Twu, Y.-W. Chen, C.-M. Shih, Preparation of silver nanoparticles using chitosan suspensions, Powder Technol. 185 (2008) 251-257.

[24] L.N. Nikitin, Yu.A. Vasil'kov, M. Banchero, L. Manna, A.V. Naumkin, V.L. Podshibikhin, S.S. Abramchuk, M.I. Buzin, A.A. Korlyukov, A.R. Khokhlov, Composite materials for medical purposes based on polyvinylpyrrolidone modified with ketoprofen and silver nanoparticles, Russ. J. Phys. Chem. A 85 (2011) 1190-1195.

[25] O.A. Belyakova, A.V. Shulenina, Ya.V. Zubavichus, A.A. Veligzhanin, A.V. Naumkin, Yu.A. Vasil'kov, Diagnostics of gold-containing surgical-dressing materials with X-ray and synchrotron radiation, J. Surf. Invest. X-ray Synchrotron Neutron Tech. 7 (2013) 509-514

[26] G. Cárdenaz, J. Díaz Visurraga, M.F. Meléndrez, C. Cruzat, A. García Cancino, Colloidal Cu nanoparticles/chitosan composite film obtained by microwave heating for food package applications, Pollym. Bull. 62 (2009) 511-514.
[27] S.I. Stoeva, A.B. Smetana, C.M. Sorensen, K.J. Klabunde, Gram-scale synthesis of aqueous gold colloids stabilized by various ligands, J. Colloid Interface Sci. 309 (2007) 94-98.

[28] C. Zhu, D. Fan, X. Ma, W. Xue, Y. Yu, Y. Luo, B. Liu, L. Chen, Effects of chitosan on properties of novel human-like collagen/chitosan hybrid vascular scaffold, J. Bioactive Compatible Polym. 76 (2009) 560-576.

[29] Patent RU2108114.

[30] I.P. Suzdalev, Yu.V. Maksimov, Yu.A. Vasil'kov, A.V. Naumkin, V.L. Podshibikhin, I.O. Volkov, Electronic and magnetic properties of Au-Fe cluster nanocomposites prepared by a sequential solvated metal atom dispersion process, Nanotechnol. Russia 3 (2008) 72-78.

[31] Yu.A. Vasil'kov, A.V. Naumkin, I.O. Volkov, V.L. Podshibikhin, G.V. Lisichkin, A.R. Khokhlov, XPS/TEM characterisation of Pt-Au/C cathode electrocatalysts prepared by metal vapour synthesis, Surf. Interface Anal. 42 (2010) 559-563.

[32] A.P. Krasnov, V.N. Aderikha, O.V. Afonicheva, V.A. Mit', N.N. Tikhonov, Yu.A. Vasil'kov, E.E. Said-Galiev, A.V. Naumkin, Yu.A. Nikolaev, Categorization system of nanofillers to polymer composites, J. Frict. Wear 31 (2010) 68-80.

[33] A.A. Chernyshev, A.A. Veligzhanin, Y.V. Zubavichus, Structural materials science end-station at the kurchatov synchrotron radiation source: recent instrumentation upgrades and experimental results, Nucl. Instrum. Methods Phys. Res. A 603 (2009) 95-98.

[34] B. Ravel, M. Newville, ATHENA, ARTEMIS, HEPHAESTUS: data analysis for X-ray absorption spectroscopy using IFEFFIT, J. Synchrotr. Rad. 12 (2005) 537-541.

[35] D.I. Svergun, Determination of the regularization parameter in indirect-transform methods using perceptual criteria, J. Appl. Crystallogr. 25 (1992) 495-503.

[36] A.I. Frenkel, A. Yevick, C. Cooper, R. Vasic, Modeling the structure and composition of nanoparticles by extended X-ray absorption fine-structure spectroscopy, Annu. Rev. Anal. Chem. 4 (2011) 23-39.

[37] C.R. Henry, Surface studies of supported model catalysts, Surf. Sci. Rep. 31 (1998) 235-325.

[38] F. Karadas, G. Ertas, E. Ozkaraoglu, S. Suzer, X-ray induced production of gold nanoparticles on $\mathrm{SiO}_{2} / \mathrm{Si}$ system and in PMMA, Langmuir 21 (2005) 437-442.

[39] R. Romand, M. Roubin, J.P. Deloume, ESCA studies of some copper and silver selenides, J. Electron Spectrosc. Relat. Phenom. 13 (1978) 229-242.

[40] G.B. Hoflund, J.F. Weaver, W.S. Epling, Ag Foil by XPS, Surf. Sci. Spectra 3 (1994) 151-156.

[41] N. Li, R. Bai, C. Liu, Enhanced and selective adsorption of mercury ions on chitosan beads grafted with polyacrylamide via surface-initiated atom transfer radical polymerization, Langmuir 21 (2005) 11780-11787.

[42] G. Beamson, D. Briggs, High Resolution XPS of Organic Polymers: The Scienta ESCA 300 Database, Wiley, Chichester, 1992. 